\title{
REGISTRO DEL PLAYERO PECTORAL CALIDRIS MELANOTOS VIEILLOT, 1819, Y NUEVAS OBSERVACIONES DE PLAYERO BLANCO CALIDRIS ALBA PALLAS, 1764, (SCOLOPACIDAE), EN MAGALLANES
}

\author{
RECORD OF THE PECTORAL SANDPIPER CALIDRIS MELANOTOS \\ VIEILLOT, 1819, AND NEW OBSERVATIONS OF THE SANDERLING \\ CALIDRIS ALBA PALLAS, 1764, (SCOLOPACIDAE), IN MAGELLAN
}

Jaime Cárcamo N. ${ }^{1,2}$ Humberto Gómez G. ${ }^{2}$ \& Patricio Barría I. ${ }^{3}$

La familia Scolopacidae está representada en Magallanes por 10 géneros que corresponden a 15 especies. El género Calidris, grupo de aves conocida comúnmente como playeros, registra 4 especies en la región, de las 18 reconocidas en el mundo (Beersma, 1996).

Las aves mencionadas de este género, originario de Norteamérica, llegan regularmente a la región de Magallanes, después de un largo viaje migratorio. Bahía Lomas en Tierra del Fuego (Chile) y Bahía San Sebastián en Argentina son los principales sitios de alimentación de estas aves en el hemisferio sur. La mayoría de las especies no superan los $26 \mathrm{~cm}$ y se alimentan principalmente en marismas o zonas barrosas de lagunas saladas, salobres o aguas dulces continentales.

Las especies registradas en Magallanes son: Calidris canutus (Linneus, 1758), Calidris alba (Pallas, 1764), Calidris fuscicollis (Vieillot, 1819) y Calidris bairdii (Coues, 1861).

Con respecto a C. alba, Humprey et al., (1970), lo califica como un visitante de verano, raro e irregular en la zona norte de Tierra del Fuego. Por otra parte Markham (1971) lo incluye en su Catalogo de los Anfibios, Reptiles, Aves y Mamíferos de la Provincia de Magallanes, en él, se señala que está presente pero de manera escasa. Venegas \& Jory (1979) informan que la especie ha sido registrada solo una vez en la región, como resultado de la colecta de un ejemplar en junio del año 1967 en una playa del sector Leñadura, al sur de Punta Arenas. Este ejemplar sería el mismo citado por Markham, y cuya piel se encuentra hoy en el Pabellón de Colecciones Biológicas "Prof. Edmundo Pisano" del Instituto de la Patagonia (UMAG). Venegas \& Sielfeld (1998) señala que no existen registros recientes y por lo tanto su status actual no estaría definido. Recientemente Couve \& Vidal (2003) y Jaramillo (2005) lo muestran en sus mapas de distribución en la misma zona norte de Tierra del Fuego, pero sin señalar sitios específicos de las observaciones.

En este trabajo se informa sobre la presencia de Calidris melanotos en la provincia de Magallanes y de C. alba, registrada en la provincia de Última Esperanza, región de Magallanes.

A la entrada norte y dentro del radio urbano de la ciudad de Punta Arenas se encuentra el humedal Tres Puentes (53 $\left.06^{\circ} 70^{\circ} 52^{\prime \prime}\right)$, con una superficie de unas 50 has., está ubicado en terrenos bajos, y es hábitat de 51 especies de aves acuáticas. Este sector es monitoreado regularmen-

\footnotetext{
${ }^{1}$ Laboratorio de Zoología, Universidad de Magallanes, jaime.carcamo@umag.cl.

2 Agrupación Ecológica Patagónica, humberto7308@gmail.com.

${ }^{3}$ Agrupación Ecológica y Ornitológica Ultima Esperanza.
} 
te por uno de los autores mediante muestreos y censos quincenales desde el año 2007 a la fecha.

Como consecuencia de las observaciones de campo regulares, el día 3 de febrero del año 2008 se registró un grupo de 6 individuos de playeros, distintos a los comúnmente observados en el humedal Tres Puentes. De este grupo, se fotografió una pareja (Fig. 1). Debido a las dudas en la identificación de la especie se envió la imagen a la Dra. Macarena Castro del Centro de Investigación de Ecosistemas de la Patagonia (CIEP), quien con posterioridad, identifico a los individuos como $C$. melanotos. Este grupo de seis aves se encontraban junto a 27 individuos de C. bairdii y C. fuscicollis. C. alba (Playero blanco), se registró en mayo de 2013. En esa oportunidad, fueron observados y fotografiados dos individuos, en la desembocadura del río Hollemberg, ubicado a $24 \mathrm{~km}$ de la ciudad de Puerto Natales, en la provincia de Última Esperanza. En este caso los ejemplares se encontraban junto a Charadrius falklandicus (Chorlo de doble collar) (Fig. 2).

\section{DISCUSIÓN Y CONCLUSIONES}

El registro de C. melanotos, marca su presencia por primera vez en la región de Magallanes. Esta especie ha sido citada para Chile por Couve \& Vidal (2003), Marín (2004) y Jaramillo (2005), con una distribución que se extiende desde el río Maule hacia el sur hasta la isla grande de Chiloé. En Argentina las observaciones están registradas hasta la Provincia de Santa Cruz (De la Peña, 1986;

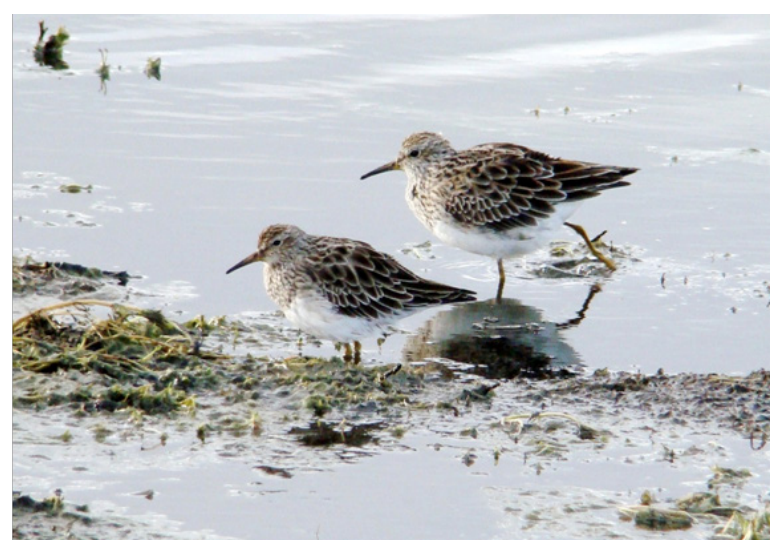

Fig. 1. Pareja de C.melanotos en Humedal Tres Puentes. (Fotografía H. Gómez)
Darrieu et al. 2008). La base pública de E-Birds muestra observaciones de la especie para Chile en la localidad de Playa Brava, Ancud, el 10 de febrero de 2012 y en Argentina, Río Deseado en noviembre del año 2004.

Con la información entregada, se consigna al Playero pectoral por primera vez en la región de Magallanes y se extiende sus registros unos 1.200 kilómetros al sur de su límite anterior mencionado por Couve \& Vidal (2003). Por ser el único registro hasta el momento, se trataría de un visitante accidental en la región.

En cuanto a $C$. alba se ratifica la existencia de la especie en la zona continental de la región de Magallanes (Markham, 1971) y no solo para el norte de Tierra del Fuego, como lo mencionan varios autores, Humprey et al., (1970), Couve \& Vidal (2003), Jaramillo (2005) y Matus (2013) com. pers. El status de la especie aún no se puede definir en la región, pero se podría presumir como un visitante ocasional fuera de la zona de Bahía Lomas. La ocurrencia de estos registros se verá fortalecida mientras se mantengan los monitoreos de avifauna en diversos sectores de la región.

\section{AGRADECIMIENTOS}

A la Dra. Macarena Castro por la confirmación de la especie C. melanotos. Al Fondo de Protección Ambiental (FPA) por la entrega de fondos para poder efectuar monitoreo de fauna en el sector humedal Tres Puentes. A Elke Shuttler, Juan Capella, Víctor Sierpe y Jorge Gibbons, por sus va-

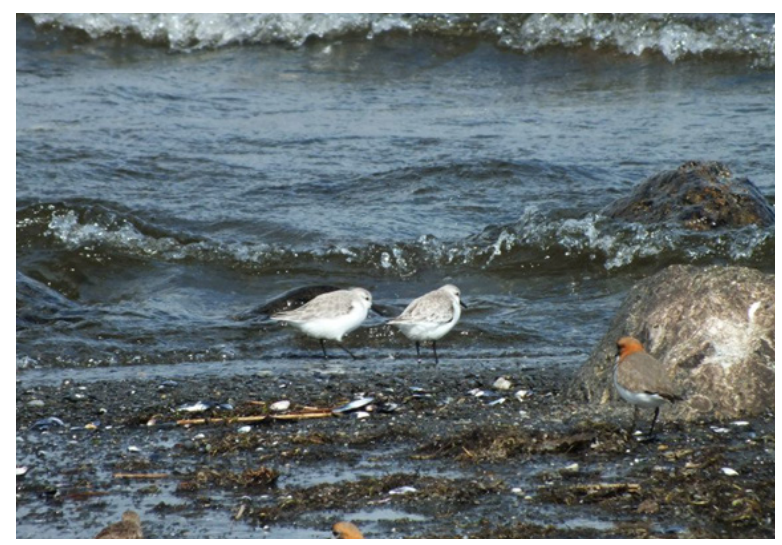

Fig. 2 C.alba en río Hollemberg, Puerto Natales. (Fotografia P. Barria) 
liosos comentarios, asimismo a un revisor anónimo por sus y críticas y sugerencias al manuscrito que permitieron mejorarlo sustancialmente.

\section{LITERATURA CITADA}

Beersma T. (1996) Family Scolapacidae (snipes, sandpipers and phalaropes). pp. 444-533 en: del Hoyo J, Elliott A y Sargatal J. (eds). Handbook of the birds of the world. Volume 3. Hoatzin to auks. Lynx Edicions, Barcelona. Couve. E. \& C. Vidal (2003). Birds of Patagonia, Tierra del Fuego \& Antartic Peninsula. Ediciones Fantástico Sur Birding. Pinting in Chile 656 pp.

De la Peña, M.R. (1986). Guía de Aves Argentinas, tomo III. GRAFOS IMPRESIONES. Santa Fe, Argentina. $80 \mathrm{pp}$.

Darrieu, C., A.R. Camperi \& S. Imberti (2008). Avifauna (Non Passeriformes) of Santa Cruz province, Patagonia (Argentina): annotated list of species. Rev. Mus. Argentino Cienc. Nat. 10 (1):111-145
E-bird Chile (2012). Base de datos. administrativos de aves y vida silvestre.

Humphrey, P.S., D. Bridge, P.W. Reynolds \& R.T. Peterson (1970). Birds of Isla Grande (Tierra del Fuego). Smithsonian Institution. Washington D.C. 411 pp.

Jaramillo. A. (2005). Aves de Chile. Lynx Ediciones. Barcelona. $240 \mathrm{pp}$.

Marín, M. (2004). Lista Comentada de las Aves de Chile. Lynx Ediciones. Barcelona. 141 pp.

Markham, B.J. (1971). Catalogo de los Anfibios, Reptiles, aves y mamíferos de la Provincia de Magallanes (Chile). Publicación del Instituto de la Patagonia, Punta Arenas. 64 pp.

Venegas, C. \& J. Jory (1979). Guía de Campo para las Aves de Magallanes. Publicaciones del Instituto de la Patagonia, Serie Monografías $\mathrm{N}^{\circ}$ 11. Punta Arenas, Chile

Venegas, C. \& W. Sielfeld (1998). Catalogo de los Vertebrados de la Región de Magallanes y Antártica Chilena. Ediciones de la Universidad de Magallanes, Punta Arenas, Chile. 122 pp. 
